\title{
Analysis of Measurement Errors in Rail Vehicles' Pantograph Inspection System
}

\author{
Slawomir Judek ${ }^{1}$, Leszek Jarzebowicz ${ }^{1}$ \\ ${ }^{I}$ Faculty of Electrical and Control Engineering, Gdansk University of Technology, \\ G. Narutowicza 11/12, 80-233 Gdansk, Poland \\ slawomir.judek@pg.gda.pl
}

\begin{abstract}
The paper presents an approach to evaluation of height measurement errors in laser scanning inspection system. The system, consisting of a laser line generator and a specialized camera, is dedicated to diagnosing carbon contact strips of railway vehicles' pantographs. While height measurement resolution is easily computable based on system parameters, determining the measurement error is troublesome due to numerous impacts. As width-to-height ratio of the scanned object is very high, typical patterns applied in references for error determination cannot be used. A set of real-case contact strips have been selected and used as patterns for determining scanning system errors. Both peak and RMS errors have been quantified to evaluate accuracy of wear estimation and defect identification, respectively.
\end{abstract}

Index Terms-Current collector; fault diagnosis; measurement by laser beam; pantograph; railway transportation.

\section{INTRODUCTION}

Routine servicing of electric rail vehicles is carried out in rolling stock depots accordingly to scheduled mileage intervals. Recently, intensive research has been conducted to complement this standard servicing with frequent condition monitoring performed by remote inspection systems [1]-[4]. Such systems, located in selected points of a railway network, aim to estimate vehicles' parameters which are crucial to safety and reliability. The parameters' monitoring is carried out on running vehicles, without any disturbance to their operation. Use of inspection systems shortens the interval before malfunction occurrence and detection. Moreover, it enables railway lines operators to have an insight into a technical condition of vehicles running within their network.

Remote inspection systems are focused either on vehicle wheels [5] or vehicle pantographs (i.e. current collectors) [6], [7]. In the latter case two targets are aimed: estimation of contact force applied by the pantograph on a contact wire or assessment of pantograph's carbon contact strips (CCSs) technical condition. CCSs are evaluated in terms of wear and hazardous damages such as a cracked or jagged surface.

This paper refers to previous research on CCSs' remote inspection. In [8] a Laser Scanning Inspection System (LSIS) for CCS diagnostics has been proposed (Fig. 1). The system uses a specialized camera and a laser line generator to acquire 3D scan of CCS surface. A specialized data processing algorithm has been applied for automatic CCS

Manuscript received 18 September, 2015; accepted 10 March, 2016. wear estimation [9]. During the research a question has arisen on the accuracy of the estimated wear value. High complexity of LSIS makes the measurement dependent on numerous factors of different nature, which makes the error analysis difficult.

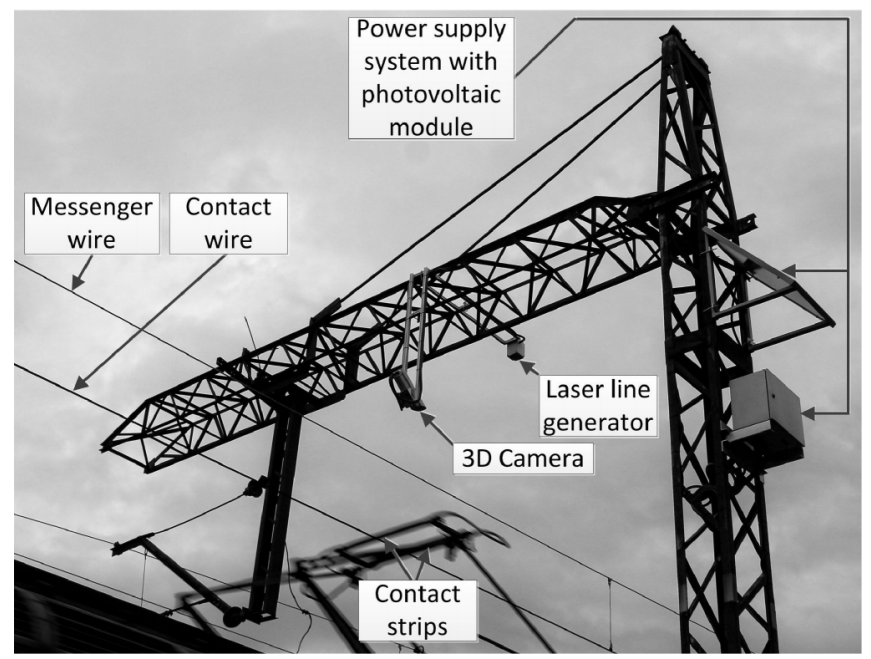

Fig. 1. Pantographs' contact strips laser scanning inspection system (LSIS) during operation.

In this paper we propose an approach to quantify errors of the CCS thickness measurement performed by the LSIS. A set of real-case CCSs is used as patterns satisfying specific requirements for LSIS error determination. Both peak and RMS errors were quantified to evaluate the accuracy of wear estimation and defect identification, respectively.

\section{LASER SCANNING INSPECTION SYSTEM}

The LSIS applies laser triangulation method to record 3dimmensional data of CCSs applied in pantographs of electric vehicles which pass by the inspection point on a railway line (Fig. 2).

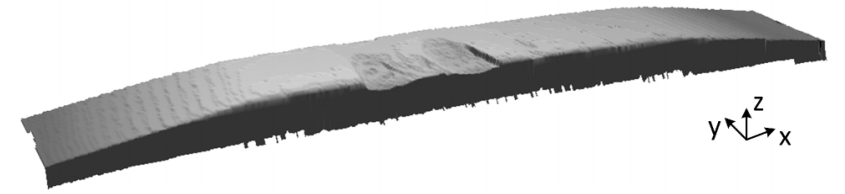

Fig. 2. 3D projection of scanning results of CCS with hazardous chips in the middle of the front edge.

The main part of the LSIS consists of a camera and a laser line generator (Fig. 3) [8]. The generator projects a light line onto the scanned CCS. The laser beam is generated 
perpendicularly to the direction of the CCS movement. The camera is installed in such a way that the light line projected on the strip can be registered at an angle of $\alpha \cong 45^{\circ}$.

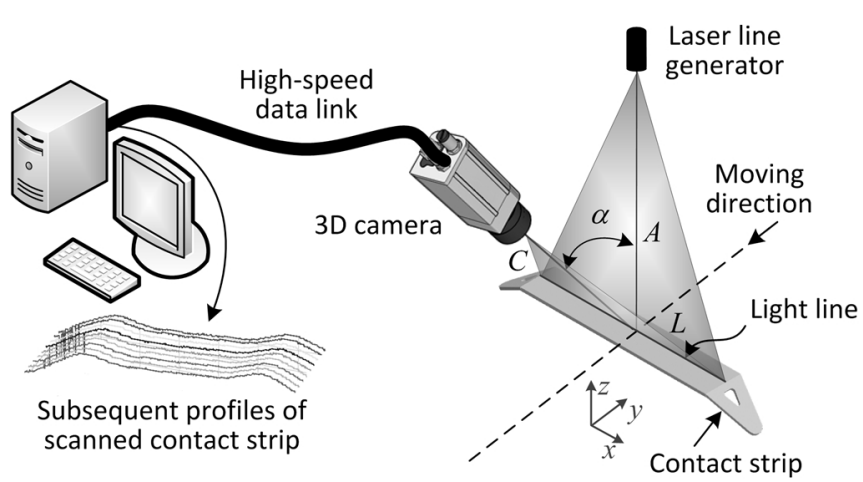

Fig. 3. Principle of laser triangulation method used in LSIS, where: $A$ laser-to-CCS distance, $C$ - camera-to-CCS distance, $L$ - length of light line.

The image of the laser line on the scanned strip in a frame recorded by the camera represents a single CCS profile $z=\mathrm{f}(x) \quad$ (Fig. 4(a)). Cyclical image registration with sufficient frame-per-second ratio and vehicle movement along $y$ axis allows for collecting a number of CCS profiles. The set of profiles forms 3-dimmensional scan $z=\mathrm{f}(x, y)$ of the CCS surface.

The parameters of camera exposure are set in a way to obtain a clear image of the light line and simultaneously to eliminate the other elements in the scene. Moreover, the region of interest is reduced to the part of the picture, where the light line may appear when projected on the CCS (Fig. 4(b)).

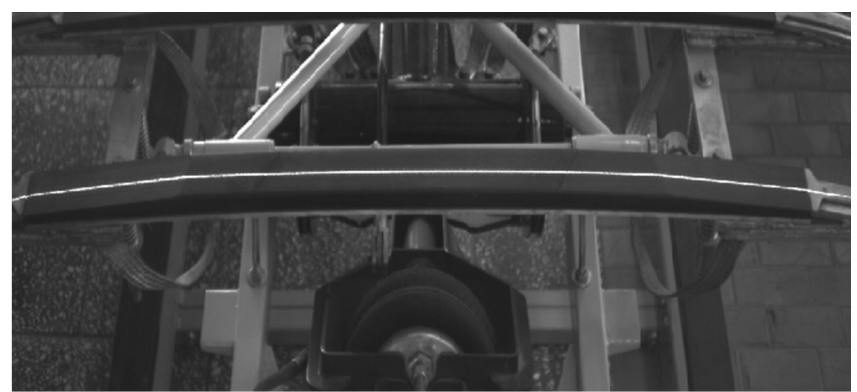

a)

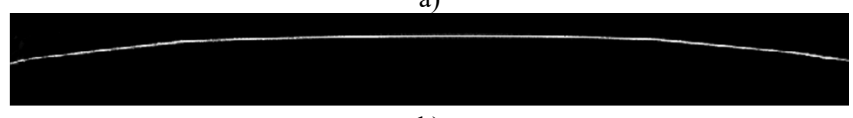

b)

Fig. 4. Image recorded by camera for temporary a) and target b) parameters of exposure and region of interest.

Extraction of the CCS profile from recorded frames requires specialised image processing. The profile $z=\mathrm{f}(x)$ is a discrete function, where height values $z_{1}, \ldots, z_{n}$ are derived for horizontal locations $x_{1}, \ldots, x_{n}$ corresponding to the particular pixel columns in the image. In each of the columns the light line is represented by several pixels (lines) of different brightness. Various processing algorithms may be applied to determine an exact pixel line corresponding to the light line position. The simplest algorithm assumes that the laser line location corresponds to the brightest pixel. However, in order to increase the resolution of height determination, more sophisticated algorithms are usually used, e.g. center-of-gravity method, which is featured by $1 / 16$ subpixel resolution [8].
The outcomes from image processing algorithm contain a light line graph in image-oriented coordinates, i.e. the CCS heights expressed in pixels. Based on spatial arrangement of the equipment ( $A, C$ and $\alpha$ marked in Fig. 3 ), as well as focal length of camera lens, the pixel coordinates are transformed into real height values representing the CCS thickness. A designated camera fitted with FPGA is used to perform image processing and geometry-related computations in real time.

LSIS applies Ranger E camera with an image sensor resolution of $1536 / 512$ pixels, and recording frequency up to 35,000 frames/s [8]. Dimensions of typical CCS and LSIS measurement resolution resulting from a designated spatial setup are presented in Table I.

TABLE I. SELECTED PARAMETERS OF CCS AND LSIS [8].

\begin{tabular}{|c|c|}
\hline Parameter & Value \\
\hline CCS typical width $(x$ axis $)$ & $1100 \mathrm{~mm}$ \\
\hline LSIS measurement resolution in $x$ axis & $0.7 \mathrm{~mm}$ \\
\hline New CCS typical thickness $(z$ axis $)$ & $15 \mathrm{~mm}$ \\
\hline Permitted CCS wear $(z$ axis) & $10 \mathrm{~mm}$ \\
\hline LSIS measurement resolution in $z$ axis & $0.5 \mathrm{~mm}$ \\
\hline CCS typical depth $(y$ axis $)$ & $60 \mathrm{~mm}$ \\
\hline LSIS measurement resolution in $y$ axis & $\begin{array}{c}\text { Depending on } \\
\text { vehicle speed }\end{array}$ \\
\hline
\end{tabular}

\section{APPROACHES TO LASER SCANNING ERROR ANALYSIS}

A common approach to improving measurement precision is to evaluate uncertainties corresponding to the measurement process and to compensate the systematic errors. When using 3D laser scanning the uncertainties are related to the camera sensor, the entire measurement process and properties of the scanned object [11]-[19].

The uncertainties related to the camera sensor can be either internal or external. The internal uncertainties concern the quality of optical equipment, i.e. lens distortion, camera focus, iris calculation etc. Isheil et al. [15] shown that the limit of optical active sensors is determined to about 2$5 \mu \mathrm{m}$, and concluded that the reduction of sensor-related uncertainties is physically limited.

The external uncertainties correspond to the position of the camera with respect to the scanned surface, the nature of the material of the scanned object like colour, absorbance, roughness, as well as the nonlinearities of the laser beam. Van Gestel et al. analysed the errors corresponding to different orientations of the camera with respect to the measured surface [12]. The results show that scan depth and incident angles (in- and out-plane) have a considerable impact on the systematic error. Vukašinović et al. studied different approaches to include surface-reflection properties into the uncertainty analysis [13]. They proved that colour is one of the most important error-influencing factors for objects having Lambertian reflectivity. Lemeš et al. investigated the relationship between ambient light intensity and the laser scanning quality [17]. The results show that influence of the light is the highest when glossy surfaces are scanned. Bračun et al. introduced comprehensive research of the properties of the light sheet curvature of laser illuminators [16]. The results show that a laser line generator emits light patterns consisting of light sheets that exhibit non-negligible bending (curvature). 


\section{Proposed Methodology of Evaluating CCS WEAR MEASUREMENT ERRORS}

The LSIS is designated for estimating CCS wear and detecting important damages. The correctness of LSIS diagnosis is affected by errors in measuring the CCS thickness. While measurement resolution is easily computable based on system parameters, determining the measurement error is troublesome. As reported in the related papers, reviewed in Section III, the error is influenced by numerous factors of different nature, e.g.: camera lens distortions, uniformity of laser line, image processing algorithms properties, geometry-related computations simplifications, absorbance of CCS surface, etc. Analytical description of these phenomena would be troublesome, thus error analysis has to be carried out experimentally.

Experimental analysis uses patterns of precisely manufactured shape and dimensions. The patterns applied in the references, e.g. ball-shaped, are dedicated to typical systems, in which the height of a scanned object is comparable to its width. The width-to-height ratio of CCSs is at a level of 50, thus evaluation of LSIS height measurement errors require dedicated patterns.

The analysis concerns two types of errors in determining $z=\mathrm{f}(x)$ profile: peak and RMS of height measurement errors. The first value is essential to damage detection algorithms, which are based on computing derivative function $\mathrm{d} z / \mathrm{d} x=\mathrm{f}(x)$ [10]. In turn, the RMS of errors corresponds to wear estimation accuracy, as minimal CCS thickness is computed using height values averaged in a relatively wide range of widths.

\section{PATTERN PREPARATION}

In order to reliably evaluate measurement errors the applied pattern should correspond to the CCS in numerous terms like: colour and roughness, as well as ranges of height and height derivative in $z=\mathrm{f}(x)$ profile. The roughness of a CCS changes during its exploitation as the factory-new strip has a relatively matte surface and it gets well-polished by a contact wire of catenary after a few thousands of kilometres of running distance. Also derivatives of height profiles $z=\mathrm{f}(x)$ differ, as new and worn strips have smooth profile, while damaged strips are featured by narrow edges.

As it is troublesome to reproduce the above discussed features in a crafted object, use of CCSs themselves as patterns is proposed. To cover all range of considered parameters three CCSs are selected: factory-new, ordinarily worn and damaged. A set of the first two consist whole possible thickness changes and light absorbance factors (matte and shiny surface). The third CCS supplements the evaluation with narrow edges corresponding to the chipped part of CCS (see Fig. 2).

Using CCSs as patterns require precise determination of their representative profiles. Therefore, a laboratory bench, shown in Fig. 5, has been constructed.

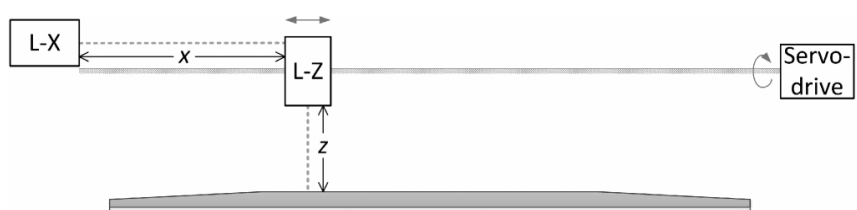

Fig. 5. Laboratory test bench for determining pattern dimensions.
The bench consists of two laser distance meters: for height $z$ measurement and for determination of horizontal position $x$. The height-measuring laser device $L-Z$ moves horizontally with a constant speed by a ball screw linear actuator driven by a speed-controlled electric servo. The uncertainty of the distance measurement was verified not to exceed $0.3 \mathrm{~mm}$, which is over three times less than the expected errors of the LSIS thickness measurement.

\section{RESUlts OF PATtERNS SCANNING}

The selected CCSs were scanned using the LSIS. The results of comparing the scanning outcomes with the pattern are shown in Fig. 6 - Fig. 8 and Table II. The new CCS, whose profile is shown in Fig. 6, has relatively uniform light absorbance at the whole width. As the matte surface is favourable for laser scanning, the errors are relatively small. The peak of individual errors is much below $1 \mathrm{~mm}$.
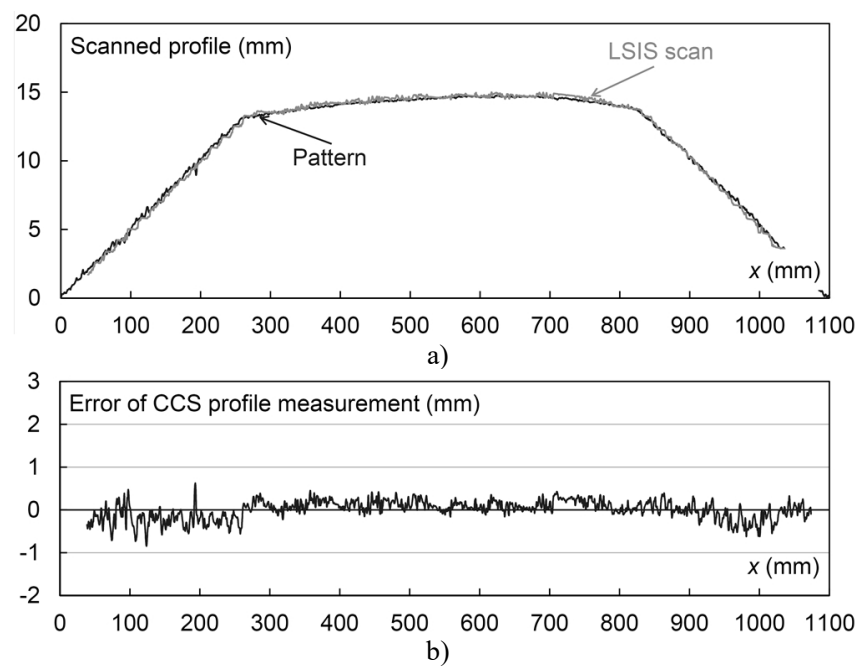

Fig. 6. Scanning results for factory-new CCS: obtained profile a) and measurement error b).

The worn CCS (Fig. 7) has a reflective surface and numerous small jags in the middle part of the profile. These properties cause unfavourable conditions for laser-based measurement, which is reflected by the quantified errors (Table II).
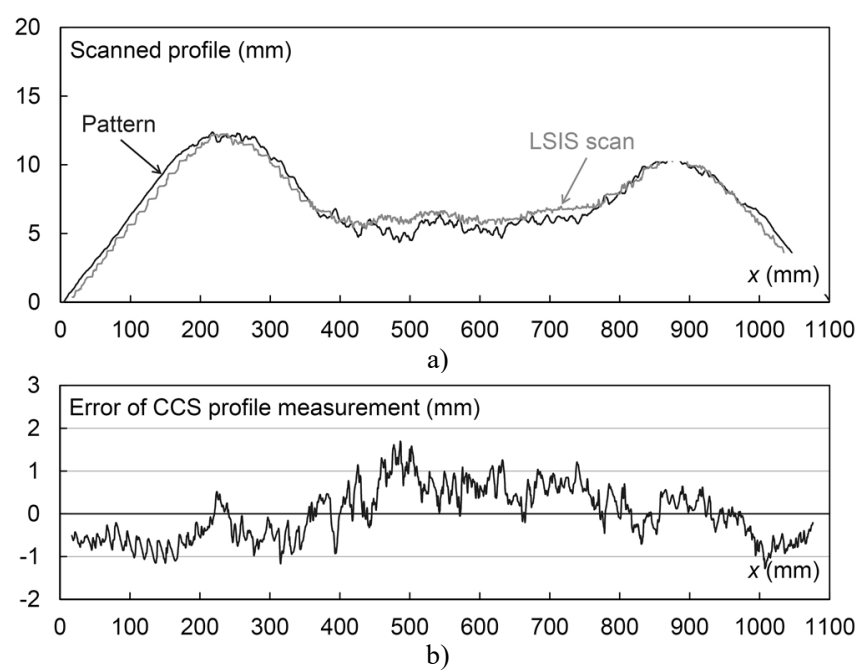

Fig. 7. Scanning results for worn CCS: obtained profile a) and measurement error b).

CCS used as the third pattern (Fig. 8) consists of chips 
featured by narrow edges. Both generic surface and the damaged area are matte. The analysis shows high peak errors at locations of narrow edges. However, this corresponds to the distortions in reconstructing $x$-axis geometry of the scanned object. Slight errors in horizontal scaling result in wrong distances between the edges. Consequently, the misaligned edges generate high peak errors. As $x$-axis oriented errors are not relevant to CCSs diagnostics, these peak errors are considered irrelevant.
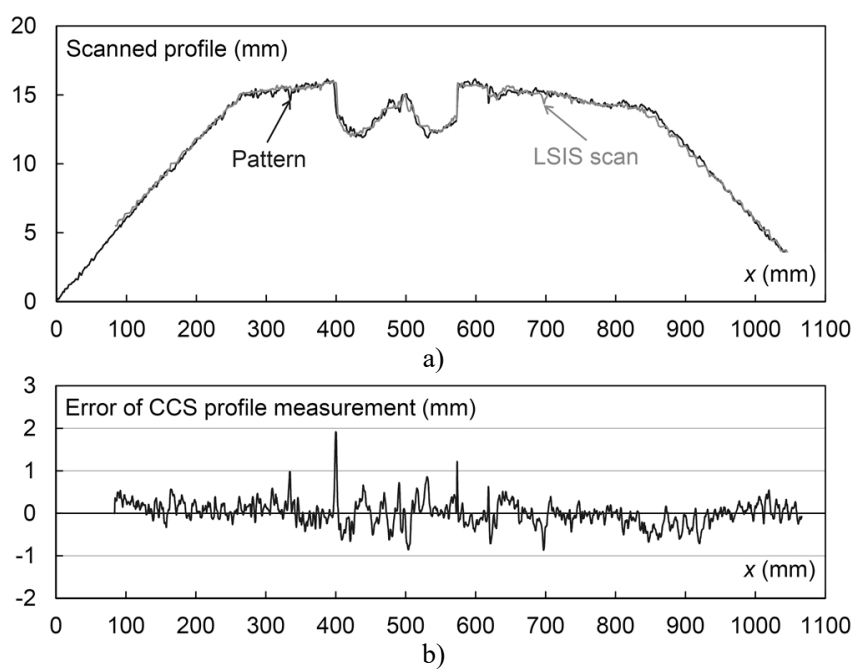

Fig. 8. Scanning results for damaged CCS: obtained profile (a) and measurement error (b).

\section{TABLE II. RESULTS OF LSIS ERROR EVALUATION}

\begin{tabular}{|c|c|c|}
\hline CCS pattern & Peak error & RMS error \\
\hline 1. Factory-new & $0.9 \mathrm{~mm}$ & $0.1 \mathrm{~mm}$ \\
\hline 2. Ordinarily worn & $1.7 \mathrm{~mm}$ & $0.4 \mathrm{~mm}$ \\
\hline 3. Damaged & $1.9 \mathrm{~mm}$ & $0.2 \mathrm{~mm}$ \\
\hline
\end{tabular}

\section{CONCLUSIONS}

In order to precisely model the varying properties of scanned objects the error analysis was carried out by using real-case CCSs patterns. The precise dimensions of these patterns were recorded using the dedicated test bench for 2dimmensional scanning.

The results of the analysis show that RMS error is kept below $0.5 \mathrm{~mm}$ in each case. This enables the LSIS to reliably estimate the CCS minimal thickness using approximately 10-level scale. Consequently, the LSIS suffices the requirements for evaluating the CCS wear. In turn, the scanned profiles are featured by relatively high noise. Peak height measurement errors are close to $2 \mathrm{~mm}$, thus detecting narrow edges by deriving $z=\mathrm{f}(x)$ profile is troublesome. Therefore, sophisticated methods of damage detection will be aimed as the future work.

\section{REFERENCES}

[1] A. Daadbin, J. Rosinski, D. Smurthwaite, "Online monitoring of essential components helps urban transport management and increases the safety of rail transport", WIT Trans. The Built Environment, vol. 128, pp. 541-552, 2012. [Online]. Available: http://dx.doi.org/ $10.2495 / \mathrm{UT} 120461$

[2] T. Maly, H. Schweinzer, M. Rumpler, "Advances in train monitoring by networked checkpoints," in Proc. IEEE Int. Workshop on Factory
Communication Systems, 2004, pp. 339-342. [Online]. Available: http://dx.doi.org/10.1109/WFCS.2004.1377741

[3] M. Bartlomiejczyk, S. Hamacek, D. Kolosov, Y. I. Zharkov, "Automated diagnostics of current pick-up disturbances in electric traction networks", in Proc. 14th Int. Conf. Environment and Electrical Engineering (EEEIC), 2014, pp. 378-381. [Online]. Available: http://dx.doi.org/10.1109/EEEIC.2014.6835897

[4] L. Makowski, A. Michalski, "Selected aspects of wireless sensors network protocol designs and their practical use [Instrumentation Notes]," IEEE Instrum. Meas. Mag., vol. 13, no. 5, pp. 45-49, 2010. [Online]. Available: http://dx.doi.org/10.1109/MIM.2010.5585074

[5] Y. Xie, M. Talbot, K. Zhao, J. Laurent, "Automatic track inspection using $3 \mathrm{~d}$ laser profilers to improve rail transit asset condition assessment and state of good repair - A preliminary study", in Transportation Research Board 93rd Annual Meeting, 2014. [Online]. Available: http://docs.trb.org/prp/14-2342.pdf

[6] M. Sacchi, S. Cagnoni, D. Spagnolettix, L. Ascariz, G. Zunino, A. Piazzi, "PAVISYS: A computer vision system for the inspection of locomotive pantographs", in Proc. Pantograph Catenary Interaction Framework for Intelligent Control, Amiens 2011.

[7] I. Aydin, M. Karakose, E. Akin, "Anomaly detection using a modified kernel-based tracking in the pantograph-catenary system", Expert Syst. Appl., vol. 42, no. 2, pp. 938-948, 2015. [Online]. Available: http://dx.doi.org/10.1016/j.eswa.2014.08.026

[8] L. Jarzebowicz, S. Judek, "3D machine vision system for inspection of contact strips in railway vehicle current collectors", in Proc. 2014 Int. Conf. Applied Electronics (AE), 2014, pp. 139-144. [Online]. Available: http://dx.doi.org/10.1109/AE.2014.7011686

[9] S. Judek, L. Jarzebowicz, "Algorithm for automatic wear estimation of railway contact strips based on 3D scanning results", in Proc. 2014 Int. Conf. Exposition on Electrical and Power Engineering (EPE), 2014, pp. 724-729. [Online]. Available: http://dx.doi.org/10.1109/ ICEPE.2014.6970004

[10] S. Judek, L. Jarzebowicz, "Wavelet transform-based approach to defect identification in railway carbon contact strips", Elektronika ir Elektrotechnika, vol. 21, no. 6, 2015. [Online]. Available: http://dx.doi.org/10.5755/j01.eee.21.6.13755

[11] D. Vala, Z. Slanina, W, Walendziuk, "Mining shaft inspection by laser photogrammetry", Elektronika ir Elektrotechnika, vol. 22, no. 1, 2016. [Online]. Available: http://dx.doi.org/10.5755/j01.eee.22. 1.14109

[12] N. Van Gestel, S. Cuypers, P. Bleys, J.-P. Kruth, "A performance evaluation test for laser line scanners on CMMs", Opt. Lasers Eng., vol. 47, no. 3-4, pp. 336-342, 2009. [Online]. Available: http://dx.doi.org/10.1016/j.optlaseng.2008.06.001

[13] N. Vukasinovic, D. Bracun, J. Mozina, J. Duhovnik, "A new method for defining the measurement-uncertainty model of $\mathrm{CNC}$ lasertriangulation scanner", Int. J. Adv. Manuf. Technol., vol. 58, no. 9-12, pp. 1097-1104, 2011. [Online]. Available: http://dx.doi.org/ 10.1007/s00170-011-3467-3

[14] A.-I. Garcia-Moreno, D.-E. Hernandez-Garcia, J.-J. GonzalezBarbosa, A. Ramirez-Pedraza, J. B. Hurtado-Ramos, F.-J. OrnelasRodriguez, "Error propagation and uncertainty analysis between 3D laser scanner and camera", Robot. Auton. Syst., vol. 62, no. 6, pp. 782-793, 2014. [Online]. Available: http://dx.doi.org/10.1016/ j.robot.2014.02.004

[15] A. Isheil, J.-P. Gonnet, D. Joannic, J.-F. Fontaine, "Systematic error correction of a 3D laser scanning measurement device", Opt. Lasers Eng., vol. 49, no. 1, pp. 16-24, 2011. [Online]. Available: http://dx.doi.org/10.1016/j.optlaseng.2010.09.006

[16] D. Bracun, M. Jezersek, J. Diaci, "Triangulation model taking into account light sheet curvature", Meas. Sci. Technol., vol. 17, no. 8, p. 2191, 2006. [Online]. Available: http://dx.doi.org/10.1088/0957$0233 / 17 / 8 / 019$

[17] S. Lemes, N. Zaimovic-Uzunovic, "Study of ambient light influence on laser 3D scanning", in Proc. 7th Int. Conf. Industrial Tools and Material Processing Technologies ICIT \& MPT, 2009, pp. 327-330.

[18] V. Knyva, M. Knyva, "Influence of 3D scanning data scattering to volume measurement of horizontal fuel tanks", Elektronika ir Elektrotechnika, vol. 20, no. 5, 2014. [Online]. Available: http://dx.doi.org/10.5755/j01.eee.20.5.7102

[19] Y. V. Chugui, A. G. Verkhoglyad, P. S. Zavyalov, E. V. Sysoev, R. V. Kulikov, I. A. Vykhristyuk, M. A. Zavyalova, A. G. Poleshchuk, V. P. Korolkov, "Optical measuring and laser technologies for scientific and industrial applications", Int. J. Autom. Technol., vol. 9, no. 5, pp. 515-524, 2015. 